\title{
CERTAIN PROPERTIES OF DERIVATIONS
}

\author{
MARK SPIVACK
}

\begin{abstract}
We consider two properties of implemented derivations on operator algebras, and give applications. One provides a simple test and leads to examples of nonimplemented derivations on commutative algebras. The other is stronger and yields a necessary and sufficient condition for derivations on $p B(H) p^{\perp}$ to be implemented, where $H$ is a Hilbert space and $p$ is a projection on $H$. Any algebra $S$ on $H$ has an extension to an algebra $S_{2}$ acting on $H \oplus \mathbf{C}$ containing such an algebra. We show that any derivation $\delta$ on an algebra $S$ is implemented if and only if $\delta$ has a bounded strongly continuous extension to $S_{2}$. If so we can construct an implementing operator explicitly.
\end{abstract}

1. Introduction. Given a bounded derivation $\delta$ on an operator algebra, the question most often asked is whether or not $\delta$ is implemented. In this paper we discuss two properties of implemented derivations, one geometrical and the other algebraic, and give some applications.

We first prove some basic results, and in particular that the first property is a consequence of the second. With the help of the geometrical property, a simple example is then constructed of a nonimplemented bounded derivation on a single rank 1 operator.

Let $B(H)$ be the set of bounded operators on a Hilbert space $H$, and let $p$ be a projection in $B(H)$. If $A$ is the commutative algebra $p B(H) p^{\perp}$, then $A^{2}=0$ and so any linear map $\delta$ from $A$ into itself is a derivation. In particular there are many nonimplemented derivations of this kind, and we give an example (again using the geometrical property). However, we can show that $\delta$ is implemented if and only if it has the algebraic property and is strongly continuous. The proof exhibits an operator which, under these conditions, implements $\delta$. This result extends to all algebras which contain $A$ and $p$ and leave $p$ invariant, and the same construction holds. Algebras of this form for some $p$ include all nontrivial nest algebras and reducible maximal triangular algebras. (It has been shown elsewhere that all derivations on nest algebras are implemented $[\mathbf{1}, \mathbf{5}$, and $\mathbf{8}]$. The differences in the proof here are discussed below.) If we extend $H$ by 1 dimension, then any algebra $S$ has a (canonical) extension to such an algebra $S_{2}$ for $p$ of rank 1 . This leads to the general result that any bounded derivation on an algebra $S$ is implemented if and only if it has a bounded, strongly continuous extension to $S_{2}$.

2. Preliminaries. Throughout, we consider operators on a Hilbert space $H$. A derivation on an operator algebra $A$ is a linear map from $A$ into $B(H)$ such that $\delta(a b)=a \delta(b)+\delta(a) b$ for all $a, b$ in $A$. $\delta$ is implemented if it is of the form $\delta(a)=b a-a b$ for all $a$ in $A$ and for some $b$ in $B(H)$. If so we say that $b$ implements $\delta$,

Received by the editors July 19, 1985 and, in revised form, November 25, 1985 and February 18, 1986.

1980 Mathematics Subject Classification (1985 Revision). Primary 47B47; Secondary 47D25. 
and we denote by $d_{b}$ the derivation thus defined. Clearly, $\delta(1)=\delta\left(1^{2}\right)=2 \delta(1)=0$. When we refer to a map on a single operator $a$ as a derivation we mean that its natural linear extension to the unital algebra generated by $a$ is a derivation. If $a$ is in $B(H)$, then $r(a), n(a)$ denote the closure of the range and the null-space of $a$ respectively. If $p$ is any projection, $p^{\perp}$ denotes its complement $1-p$. (When we refer to projections we always have in mind selfadjoint projections.) If $A$ is any set of operators, we denote by $\operatorname{lat}(A)$ the lattice of subspaces left invariant by $A$. If $L$ is any set of projections, the algebra of operators leaving every element of $L$ invariant is denoted by $\operatorname{alg}(L)$.

A nest on $H$ is a totally-ordered strongly closed set of projections on $H$ containing 0 and 1. A nest algebra $N$ on $H$ is then a set $\operatorname{alg}(L)$ of all operators leaving invariant every element of a nest $L$ (see [6]). It follows that $\operatorname{lat}(N)=L$. An algebra $S$ is triangular if $S \cap S^{*}$ is maximal Abelian in $B(H)$ (see [4]). Then $S \cap S^{*}$ is called the diagonal of $S$. Since any triangular algebra containing $S$ has the same diagonal, $S$ is contained in some maximal triangular algebra. $S$ is called reducible if lat $(S)$ is nontrivial, and irreducible otherwise. The following property is also common to nest and maximal triangular algebras.

LEMMA $1[\mathbf{4}, \mathbf{6}]$. Let $A$ be a nest algebra or a maximal triangular algebra, and let $p$ be a projection in lat $(A)$. Then $A$ contains the set $p B(H) p^{\perp}$.

It follows, in particular, that if $\xi$ is any nonzero vector in $n(p)$ and $\eta$ is in $r(p)$, then the rank 1 operator $a=\xi \otimes \eta$ is in $A$.

We come now to the main definitions.

Definition 1. A derivation $\delta$ on an algebra $A$ has Property $G$ if $\delta(a) \xi$ is in $r(a)$ whenever $\xi$ is in $n(a)$, for all $a$ in $A$.

DEFinition 2. A derivation $\delta$ on $A$ has Property $D$ if

$$
\delta(a b c)=\delta(a b) c+a \delta(b c)-a \delta(b) c
$$

whenever $b$ is in $A$, and $a b, b c$, and $a b c$ are also in $A$. $\delta$ has Property $D_{p}$ if (1) holds when $b$ is a partial isometry, and Property $D_{1}$ if (1) holds when $b$ is of rank 1 .

If every bounded derivation on an algebra $A$ has one of these properties, we say that $A$ has that property.

As already mentioned, Property $D$ is the stronger condition. When we know that it holds we can, in effect, use operators outside the domain of a derivation to examine its behavior.

3. Basic results. Let $\delta$ be an implemented derivation. Then it follows immediately from the definition that $\delta$ obeys Property $G$. Since $\delta$ is implemented it may be extended to the whole of $B(H)$, and a simple application of the derivation law yields Property $D$ :

$$
\delta(a b c)=\delta(a b) c+a b \delta(c)=\delta(a b) c+a \delta(b c)-a \delta(b) c .
$$

This of course has no meaning unless $\delta$ is defined on $c$.

If $p$ is any projection it is easily shown that $\delta(p) p^{\perp}=p \delta(p)$, which is just Property $G$. In fact we have the following, which we will not prove.

LEMMA 2. A map $\delta$ on a projection acts as a derivation if and only if it obeys Property $G$. 
It is well known that any derivation on a projection is implemented, and this follows from the same fact. (One such implementing operator is given by $(1-2 p) \delta(p)$.)

\section{Lemma 3. Property $D$ implies Property $G$.}

Proof. Let $\delta$ have Property $D$. Let $a$ be in the domain $A$ of $\delta$, and let $p$ and $q$ be the projections onto $r(a)$ and the complement of $n(a)$ respectively. Then $a=p a=a q=p a q$. So $\delta(a)=\delta(p a) q+p \delta(a q)-p \delta(a) q$, and the result follows.

We briefly mention here derivations on von Neumann algebras. Such derivations are automatically continuous [7]. It has been shown that derivations on almost all classifications of von Neumann algebras are implemented (see Christensen [2]).

LeMma 4. Any von Neumann algebra $A$ has Property $D$.

Proof. Let $b, a b, b c$, and $a b c$ be in $A$ and let $q$ be the projection onto $n(b)^{\perp}$. Then $q c$ is in $A$. So

$$
\begin{aligned}
\delta(a b c) & =\delta(a b) q c+a b \delta(q c)=\delta(a b) c-a b \delta(q) c+a b \delta(q c) \\
& =\delta(a b) c-a \delta(b) c+a \delta(b) q c+a b \delta(q c) \\
& =\delta(a b) c-a \delta(b) c+a \delta(b c)
\end{aligned}
$$

as required.

\section{Further results and applications.}

4.1. A simple nonimplemented derivation. In the study of certain operator algebras the question naturally arises: Is a derivation on a single rank 1 operator automatically implemented? Below, with the help of Property $G$, we give a very simple example in which this does not hold.

Let $H$ be any Hilbert space of dimension 3 or greater. Let $\xi, \eta$ be orthogonal unit vectors in $H$, and denote by $q$ the rank 1 operator $\xi \otimes \eta$. Notice that $q^{2}=0$. Let $A$ be the unital algebra generated by $q$. Then $A=\{\alpha+\beta q: \alpha, \beta \in \mathbf{C}\}$, and $A$ is nonselfadjoint. We define a derivation on $A$ as follows: Put $\delta(q) \xi=-\xi$, and $\delta(q) \zeta=\zeta$ whenever $\xi_{\perp} \zeta$. Now extend by linearity to the whole of $A$.

It is obvious that $\delta$ is well defined. To prove that $\delta$ obeys the derivation rule we need only show that $q \delta(q)+\delta(q) q=\delta\left(q^{2}\right)=0$, and this is almost trivial. Hence $\delta$ is a derivation. Finally, Property $G$ fails since, for $\zeta$ orthogonal to both $\xi$ and $\eta$ we have $\varsigma \in n(q), \zeta \in r(q)$, and $\delta(q) \varsigma=\varsigma$. Thus $\delta$ cannot be implemented.

In this special case Properties $D$ and $G$ are actually equivalent. This is because if, for example, $a q \in A$, then $a q$ is a scalar multiple of $q$.

$A$ is the simplest commutative nonselfadjoint algebra that can be defined. In the next section we give an example at the other extreme, for a maximal commutative nonselfadjoint algebra. We mention that Gilfeather [3] has also produced an example of a nonimplemented derivation on a nonselfadjoint algebra.

As a point of interest we give (without proof) an example of a natural unbounded derivation for which Property $G$ fails: Let $H$ be any infinite dimensional Hilbert space. Choose $a$ in $B(H)$ such that $n(a)$ is not contained in $r(a)$ and such that $\left\{a^{n}: n \geq 0\right\}$ is linearly independent (where $a^{0}=1$ ). The equation $\delta\left(a^{n}\right)=n a^{n-1}$ gives rise to a "differentiation" on the unital algebra generated by $a$. This is a well-defined unbounded derivation for which, as we have said, Property $G$ does not hold. 
4.2. Derivations on algebras containing $p B(H) p^{\perp}$. Let $p$ be a nontrivial projection and let $A$ be the algebra $p B(H) p^{\perp}$. Suppose that $\delta: A \rightarrow A$ is a bounded linear map. Then, as mentioned above, $\delta$ is a derivation since it automatically obeys the derivation rule. In the absence of a multiplicative structure it is of interest to find minimal conditions for implementation to be automatic. Note that $\delta$ extends trivially to the unital algebra $A_{1}$ generated by $A$, which is maximal commutative.

As an example of a nonimplemented derivation on $A$ consider the following: Let $\xi_{0}, \xi_{1}$ be orthogonal unit vectors in $n(p)$, and let $\eta_{0}, \eta_{1}$ be orthogonal unit vectors in $r(p)$. If $v=\xi_{0} \otimes \xi_{1}$, and $u=\eta_{1} \otimes \eta_{0}$, then $\delta(a)=u a v$ defines a bounded derivation on $A$. However, if $a$ is given by $\xi_{1} \otimes \eta_{1}$, then $\delta(a) \xi_{0}=\eta_{0}$. So Property $G$ fails and $\delta$ cannot be implemented. Notice, incidentally, that the identity operator on $A$ is implemented, for example by the projection $p$.

Note that if $\delta_{1}$ is a derivation defined on an algebra containing $A$ and $p$, then we can assume that its restriction to $A$ maps $A$ into itself: There exists an operator $b$ such that $\delta_{1}(p)=d_{b}(p)$. Replace $\delta_{1}$ by $\delta=\delta_{1}-d_{b}$. Then $\delta(p)=0$, and $\delta$ is implemented if and only if $\delta_{1}$ is implemented. Also

$$
\delta\left(p a p^{\perp}\right)=\delta(p) a p^{\perp}+p \delta(a) p^{\perp}+p a \delta\left(p^{\perp}\right)=p \delta(a) p^{\perp} .
$$

We can now proceed with the construction. Note that for a rank 1 operator $a=\xi \otimes \eta, a^{*}$ is just $\eta \otimes \xi$. Choose fixed unit vectors $\xi_{0} \in n(p)$ and $\eta_{0} \in r(p)$, and write $p_{0}=\xi_{0} \otimes \xi_{0}$ and $q_{0}=\xi_{0} \otimes \eta_{0}$. If $\xi$ is any unit vector in $n(p)$, put $p_{\xi}=\xi \otimes \xi$. We define an operator $b=p b p+p^{\perp} b p^{\perp}$ (so that $b$ commutes with $p$ ) as follows:

$$
b \eta=b a \xi_{0}=\delta\left(a p_{0}\right) \xi_{0}
$$

for any $a \in A$ such that $a \xi_{0}=\eta$, and, if $\xi$ is in $n(p)$ and $q_{1}=\xi \otimes \eta_{0}$,

$$
p_{\xi} b=p_{\xi} b p^{\perp}=-q_{1}^{*} \delta\left(q_{1}\right)+q_{1}^{*} \delta\left(q_{0}\right) q_{0}^{*} q_{1} .
$$

It is not immediately clear that $b$ is in $B(H)$.

LEMMA 5. $b$ is a well-defined bounded linear operator.

PROOF. Consider separately the parts $b_{1}=p b p$ and $b_{2}=p^{\perp} b p^{\perp}$ of $b . b_{1}$ is clearly well defined and linear, and is bounded because

$$
\left\|b_{1} a \xi_{0}\right\|=\left\|\delta\left(a p_{0}\right) \xi_{0}\right\| \leq\|\delta\|\left\|a p_{0}\right\|=\|\delta\|\left\|a \xi_{0}\right\| .
$$

Similarly it is clear that $b_{2}$ is well defined and bounded. We must show that it is linear. For this it is sufficient to show that $b_{2}^{*}$ is linear: Let $\xi$ be a unit vector in $n(p)$, and let $q_{1}=\xi \otimes \eta_{0}$. Then

$$
b_{2}^{*} \xi=-\delta\left(q_{1}\right)^{*} q_{1} \xi+q_{1}^{*} q_{0} \delta\left(q_{0}\right)^{*} q_{1} \xi
$$

and it is clear that this is linear in $\xi$.

THEOREM 1. $b$ implements the derivation $\delta$ on $A$ if and only if $\delta$ is strongly continuous and has Property $D_{1}$.

PROOF. It need only be shown that if $\delta$ has these properties then $b$ implements $\delta$. If we can show that $\delta(q)=d_{b}(q)$ for all rank 1 operators $q$ in $A$ then the result will follow, by strong continuity. So let $\xi_{0}, \eta_{0}$, and $q_{0}$ be as above. Let $q=\xi \otimes \eta$ 
be any rank 1 operator in $A$, where $\xi, \eta$ are of norm 1 . Define, in addition, the rank 1 operators

$$
q_{1}=\xi \otimes \eta_{0}, \quad q_{2}=\xi_{0} \otimes \eta, \quad \text { and } \quad p_{1}=\xi \otimes \xi .
$$

Then we have $q=q q_{1}^{*} q_{0} q_{0}^{*} q_{1}$ and $q_{2}=q q_{1}^{*} q_{0}$. First suppose that $\zeta$ is in $r(p)$. Then $(b q-q b)_{\zeta}=-q b_{1} \zeta=0=\delta(q) \zeta$. Now let $\zeta$ be in $n(p)$. Then

$$
\begin{aligned}
(b q-q b) \varsigma & =b_{1} q \varsigma-q p_{1} b \varsigma \\
& =\left[\delta\left(q_{2}\right) q_{0}^{*} q_{1}+q q_{1}^{*} \delta\left(q_{1}\right)-q q_{1}^{*} \delta\left(q_{0}\right) q_{0}^{*} q_{1}\right] \varsigma \\
& =\left[\delta\left(q q_{1}^{*} q_{0}\right) q_{0}^{*} q_{1}+q q_{1}^{*} \delta\left(q_{1}\right)-q q_{1}^{*} \delta\left(q_{0}\right) q_{0}^{*} q_{1}\right] \zeta .
\end{aligned}
$$

However Property $D_{1}$ says that this is just $\delta(q) \zeta$, as required.

Now let $S$ be any algebra containing $A$ and $p$, and which leaves $p$ invariant. This holds, for example, if $S$ is a nest algebra or a maximal triangular algebra such that $p \in \operatorname{lat}(S)$. Let $\delta: S \rightarrow B(H)$ be a bounded derivation.

COROLlaRY 1. $\delta$ is implemented if and only if its restriction to $A$ is strongly continuous and satisfies Property $D_{1}$. If so, then $b$ implements $\delta$ on $S$.

PROOF. Suppose that $\delta$ has the stated properties. (As before they are automatically true if $\delta$ is implemented.) As already mentioned we can assume that $\delta(p)=0$, and therefore that the restriction of $\delta$ to $A$ maps $A$ into itself. We consider several cases. Recall that $b$ commutes with $p$. First let $a=p a p$. Suppose that $\xi \in n(p)$. Then

$$
d_{b}(a) \xi=(b a-a b) p^{\perp} \xi=0
$$

and $\delta(a) \xi=\delta(a) p^{\perp} \xi=\left[\delta\left(a p^{\perp}\right)-a \delta\left(p^{\perp}\right)\right] \xi=0$. On the other hand, if $\xi \in r(p)$, and if $c \xi_{0}=\xi$ then

$$
\begin{aligned}
(b a-a b) \xi & =(b a c-a b c) \xi_{0}=\left[\delta\left(a c p_{0}\right)-a \delta\left(c p_{0}\right)\right] \xi_{0} \\
& =\delta(a) c \xi_{0}=\delta(a) \xi .
\end{aligned}
$$

Now let $a=p^{\perp} a p^{\perp}$. If $\xi \in r(p)$ then, again, $d_{b}(a) \xi=\delta(a) \xi=0$. Suppose now that $\xi \in n(p)$. Then $p(b a-a b) \xi=p \delta(a) \xi=0$. Consider, finally, $p^{\perp} \delta(a)$ : Choose a basis $\left\{\xi_{\alpha}\right\}$ for $n(p)$. Let $\eta_{0}$ be as in Theorem 1, and put $p_{\alpha}=\xi_{\alpha} \otimes \xi_{\alpha}$ and $q_{\alpha}=\xi_{\alpha} \otimes \eta_{0}$. Define the constants $r_{\alpha \beta}$ in $\mathbf{C}$ by $p_{\alpha} a p_{\beta}=r_{\alpha \beta} q_{\alpha}^{*} q_{\beta}$, where $\xi_{\alpha}, \xi_{\beta}$ are two basis vectors. Then

$$
\begin{aligned}
p_{\alpha}(b a & -a b) \xi=q_{\alpha}^{*} \delta\left(q_{0}\right) q_{0}^{*} q_{\alpha} a p^{\perp} \xi-q_{\alpha}^{*} \delta\left(q_{\alpha}\right) a \xi-\sum_{\beta} p_{\alpha} p^{\perp} a p_{\beta} b \xi \\
& =-q_{\alpha}^{*} \delta\left(q_{\alpha}\right) a \xi+\sum_{\beta}\left[q_{\alpha}^{*} \delta\left(q_{0}\right) q_{0}^{*} q_{\alpha} a p_{\beta}-p_{\alpha} a q_{\beta}^{*} \delta\left(q_{0}\right) q_{0}^{*} q_{\beta}+p_{\alpha} a q_{\beta}^{*} \delta\left(q_{\beta}\right)\right] \xi \\
& =-q_{\alpha}^{*} \delta\left(q_{\alpha}\right) a \xi+\sum_{\beta}\left[r_{\alpha \beta} q_{\alpha}^{*} \delta\left(q_{0}\right) q_{0}^{*} q_{\beta}-r_{\alpha \beta} q_{\alpha}^{*} \delta\left(q_{0}\right) q_{0}^{*} q_{\beta}+r_{\alpha \beta} q_{\alpha}^{*} \delta\left(q_{\beta}\right)\right] \xi \\
& =-q_{\alpha}^{*} \delta\left(q_{\alpha}\right) a \xi+\sum_{\beta} q_{\alpha}^{*} \delta\left(r_{\alpha \beta} q_{\beta}\right) \xi=-q_{\alpha}^{*} \delta\left(q_{\alpha}\right) a \xi+\sum_{\beta} q_{\alpha}^{*} \delta\left(q_{\alpha} a p_{\beta}\right) \xi \\
& =-q_{\alpha}^{*} \delta\left(q_{\alpha}\right) a \xi+q_{\alpha}^{*} \delta\left(q_{\alpha} a\right) \xi=q_{\alpha}^{*} q_{\alpha} \delta(a) \xi=p_{\alpha} \delta(a) \xi .
\end{aligned}
$$

Hence for all $\alpha, p_{\alpha} d_{b}(a) \xi=p_{\alpha} \delta(a) \xi$, and the proof is complete.

In particular, since it is known that every derivation on a nest algebra is implemented $[\mathbf{1}, \mathbf{5}$, and $\mathbf{8}]$ we have the following corollary. 
COROllaRY 2. If $S$ is any nontrivial nest algebra and $\delta: S \rightarrow B(H)$ is a derivation, then $b$, defined as above for any projection $p$ in lat $(S)$, implements $\delta$.

The surprising feature of the construction of the operator $b$ is that it involves very little knowledge of the algebra $S$. It is this which distinguishes the proof for nest algebras from previous ones, which depend on a limiting procedure and more detailed knowledge of the underlying nest.

Property $D$ (or $D_{1}$ ) would be useless if it were not possible to test whether it holds in individual cases. However this can be done directly, for example, for von Neumann algebras (Lemma 4) and nest algebras (although for brevity we have not done so here).

4.3. Extensions of derivations. Suppose that the algebras $A$ and $S$ are as above, where the projection $p$, or $p^{\perp}$, is now of rank 1. Let $\delta: S \rightarrow B(H)$ be a derivation.

Lemma 6. The restriction of $\delta$ to $A$ has Property $D$.

Proof. This is immediate. For example, let $p$ be of rank 1. Let $b$ be in $A$ such that $a b, b c$, and $a b c$ are also in $A$. Again we can assume that $\delta$ maps $A$ into itself. Now $a p=\alpha p$ for some $\alpha \in \mathbf{C}$, so

$$
\begin{aligned}
\delta(a b) c+a \delta(b c)-a \delta(b) c & =\alpha[\delta(b) c+\delta(b c)-\delta(b) c] \\
& =\alpha \delta(b c)=\delta(a b c) .
\end{aligned}
$$

Now let $S$ be any unital algebra, and let $\delta: S \rightarrow B(H)$ be a derivation. Put $H_{2}=H \oplus H_{1}$, where $H_{1}$ is just a copy of $\mathbf{C}$, and let $p$ denote the rank 1 projection of $H_{2}$ onto $H_{1}$. Then the set $S_{2}=S+p B\left(H_{2}\right) p^{\perp}+\mathrm{C} p$ is a unital algebra extending $S$ to $H_{2}$. (Note that $p B\left(H_{2}\right) p^{\perp}$ is, as a vector space, simply a copy of $H$.)

THEOREM 2. $\delta$ is implemented on $S$ if and only if it has a bounded, strongly continuous extension to $S_{2}$.

PROOF. It is immediate from Corollary 1 and Lemma 6 that if the extension exists, then it is implemented on $S_{2}$ by some operator $b_{2}$ in $B\left(H_{2}\right)$, where $b_{2}$ commutes with $p$. Thus $b_{2} p^{\perp}$ implements $\delta$ on $S$.

It turns out that in certain cases it is not too difficult to find extensions of this type. The possibilities raised by this technique will be discussed in another paper.

ACKNOWLEDGMENTS. I would like to thank the referee for some very helpful suggestions, including the present form of Lemma 4 and its proof. Part of this work arose during the preparation of my Ph.D. thesis at Manchester University, with the financial support of the Science Research Council, and as a teaching assistant at Tel-Aviv University. I am most grateful to E. C. Lance, R. Plymen, B. Solel, and A. Lazar for various discussions on the work.

\section{REFERENCES}

1. E. Christensen, Derivations of nest algebras, Math. Ann. 229 (1977), 151-161.

2. __ Extensions of derivations, J. Funct. Anal. 27 (1978), 234-247.

3. F. Gilfeather, Derivations on certain CSL algebras, J. Operator Theory 11 (1984), 145156.

4. R. V. Kadison and I. M. Singer, Triangular operator algebras, Amer. J. Math. 82 (1960), 227-259.

5. E. C. Lance, Cohomology and perturbations of nest algebras, Proc. London Math. Soc. 43 (1981), 334-356. 
6. J. R. Ringrose, On some algebras of operators, Proc. London Math. Soc. 15 (1965), 61-83. 7. _ Automatic continuity of derivations on operator algebras, J. London Math. Soc. 5 (1972), 432-438.

8. M. Spivack, Derivations and nest algebras on Banach space, Israel J. Math. 50 (1985), 193-200.

Department of Applied Mathematics and Theoretical Physics, University of CAMBRIDGe, CAMBRIDGe CB3 9EW, UNited Kingdom 